\title{
Ulinastatin inhibits renal tubular epithelial apoptosis and interstitial fibrosis in rats with unilateral ureteral obstruction
}

\author{
QING-FANG ZHANG
}

\begin{abstract}
Department of Nephrology, Second Affiliated Hospital of Harbin Medical University, Harbin, Heilongjiang 150086, P.R. China
\end{abstract}

Received April 15, 2017; Accepted September 5, 2017

DOI: $10.3892 / \mathrm{mmr} .2017 .7692$

\begin{abstract}
The effect of ulinastatin (UTI) on renal tubular epithelial apoptosis and interstitial fibrosis in rats with unilateral ureteral obstruction (UUO) was investigated. A total of 18 male Wistar rats were randomly divided into the following 3 groups: The Sham group $(n=6)$, the UUO group ( $n=6)$, and the UTI group ( $n=6)$. In the UUO and UTI groups, the left ureter was ligated to establish a UUO model. Starting from day 1 after surgery, an intervention treatment was performed using normal saline $(1 \mathrm{ml} / \mathrm{kg} / \mathrm{d})$ and UTI $(40,000$ unit $/ \mathrm{kg} / \mathrm{d})$. On day 7 after surgery, 6 rats from each group were sacrificed. In the Sham group, the left ureter was only freed, not ligated; after 7 days of abdominal closure, all of the rats were sacrificed. Blood samples were collected prior to sacrificing the animals to measure the blood urea nitrogen (BUN) and serum creatinine (Scr). The incidence of renal interstitial lesions on the obstruction side was observed by hematoxylin and eosin, and Masson staining. Terminal deoxynucleotidyl transferase dUTP nick end labeling (TUNEL) staining, and immunohistochemical detection of apoptosis regulator $\mathrm{Bax}$ ( $\mathrm{Bax}$ ), apoptosis regulator Bcl-2 (Bcl-2) and caspase-3 were performed to observe the presence of renal tubular epithelial cell apoptosis. The UTI did not have a significant influence on the mouse BUN and Scr levels in any of the groups $(\mathrm{P}>0.05)$. Compared with that in the Sham group, renal tissue injury in the UUO group was significantly aggravated with renal tubular dilation, epithelial cell atrophy, renal interstitial inflammatory cell infiltration and fibrous tissue hyperplasia $(\mathrm{P}<0.01)$. Furthermore, the renal tubular epithelial TUNEL+ cell number and Bax and caspase- 3 levels were increased, and the expression of $\mathrm{Bcl}-2$ was decreased $(\mathrm{P}<0.01)$. Following the UTI treatment, the renal interstitial injury at the obstruction
\end{abstract}

Correspondence to: Dr Qing-Fang Zhang, Department of Nephrology, Second Affiliated Hospital of Harbin Medical University, 246 Xuefu Road, Nangang, Harbin, Heilongjiang 150086, P.R. China

E-mail: zqfhlj@aliyun.com

Key words: ulinastatin, UUO, apoptosis, fibrosis side was significantly attenuated $(\mathrm{P}<0.05)$, the renal tubular epithelial TUNEL+ cell number, and Bax and caspase-3 levels significantly decreased, and the expression of $\mathrm{Bcl}-2$ was restored $(\mathrm{P}<0.05)$. UTI inhibited renal tubular epithelial apoptosis and interstitial fibrosis in UUO rats.

\section{Introduction}

Ureteral obstruction is a common urological disease. If not treated in a timely manner, it will progress toward renal interstitial fibrosis, which is a common, irreversible pathological change that is also observed in other chronic and progressive kidney diseases (1). The unilateral ureteral obstruction (UUO) model is a classical animal model used to study obstructive renal tubular epithelial apoptosis and interstitial fibrosis (2). The major presentations of renal injury caused by UUO are renal tubular epithelial cell apoptosis, interstitial inflammatory reaction and progressive fibrosis, of which, cell apoptosis has a close relationship with renal fibrosis (3). Inhibition of cell apoptosis may delay or reverse renal tubular interstitial fibrosis to improve renal function and the prognosis of patients with kidney diseases (4). The Bcl-2 family members are key factors in the regulation of apoptosis. Bcl-2 is a proto-oncogene and can inhibit cell apoptosis, whereas Bax promotes cell apoptosis. Up-regulation of Bcl-2 and downregulation of Bax indicates that renal cell apoptosis is diminishing (5). During cell apoptosis, the caspase family member, caspase-3, is the most important terminal cleavage enzyme. It plays the role of an apoptosis executor and is also an important component in the killing mechanism of cytotoxic T lymphocytes (CTL).

Ulinastatin (UTI) is a type of glycoprotein isolated from human urine. It is a typical Kunitz-type, broad-spectrum, high-efficiency protease inhibitor (6). It functions in the clearance of oxygen free radicals and the inhibition of inflammatory reactions $(7,8)$. It is applied clinically for the treatment of acute pancreatitis and extracorporeal circulation injury and the prevention of shock and surgical invasion. Recent studies indicate that UTI inhibited cell apoptosis during brain injury $(9,10)$ and had a protective function in various acute renal injuries $(11,12)$. In addition, it has been reported that UTI inhibited lung (13) and renal (14) fibrosis; however, the specific mechanism is still not clear. This study aimed to investigate whether UTI inhibits renal interstitial fibrosis by preventing renal cell apoptosis in UUO rats. 


\section{Materials and methods}

Ethics statement and animals. Male Wistar rats aged 8-12 weeks with body weights of 180-200 g were provided by the Animal Experimental Center of the Second Affiliated Hospital of Harbin Medical University (Harbin, China). All rats were nurtured and maintained according to the "Care and Use of Laboratory Animals' guidelines published by the National Institute of Health (15). The animal use protocol was reviewed and approved by the Institutional Animal Care and Use Committee (IACUC) of the Second Affiliated Hospital of Harbin Medical University.

Establishment of the animal model and grouping. A total of 18 male Wistar rats were randomly divided into the following 3 groups: The sham surgery group (Sham, $n=6$ ), the UUO group (UUO, n=6) and the UTI group (UTI, n=6). The UUO model was established according to previous literature (16). The UUO and UTI groups received a left ureteral ligation after intraperitoneal anesthesia using $10 \%$ chloral hydrate and received intervention using normal saline $(1 \mathrm{ml} / \mathrm{kg} / \mathrm{d})$ and UTI (40,000 unit/kg/d; Techpool Bio-pharma Co., Ltd., Guangdong, China) starting on day 1 after surgery. On day 7 after surgery, the rats were sacrificed using euthanasia. In the Sham group, the left ureter was freed but not ligated after intraperitoneal anesthesia using $10 \%$ chloral hydrate; after 7 days of abdominal closure, the rats were sacrificed using euthanasia.

Specimen collection and processing. Before the rats were sacrificed, $5 \mathrm{ml}$ of venous blood was collected from each rat. The serum samples were separated by centrifugation and stored in a $-20^{\circ} \mathrm{C}$ freezer. Normal saline was perfused through the left ventricle to wash the renal tissues. The renal cortical tissues at the obstruction side were collected; some tissues were placed into cryotubes, snap frozen and stored in a $-80^{\circ} \mathrm{C}$ freezer. The remaining tissues were divided into 2 blocks, each with a size of approximately $4 \times 5 \mathrm{~mm}$; fixed in $4 \%$ paraformaldehyde for $36 \mathrm{~h}$; and conventionally prepared in sections of approximately $4-\mu \mathrm{m}$ thickness for pathology staining, immunohistochemistry and terminal deoxynucleotidyl transferase dUTP nick end labeling (TUNEL) staining.

Detection of biochemical indicators. The serum blood urea nitrogen (BUN) and serum creatinine (Scr) were detected using an automatic biochemical analyzer.

Examination of pathological changes in renal tissues using renal histopathology and semi-quantitative analysis. Paraffin sections were used for routine hematoxylin and eosin (H\&E) and Masson staining. The levels of renal tubular injury and renal interstitial fibrosis were evaluated using a semi-quantitative scoring method (17). Twenty-five different high-power fields were randomly selected in each specimen (magnification, x100). Renal tubular injury included vacuolar degeneration, necrosis and renal tubular dilation and atrophy. Renal tubular injury classification was as follows: 0 points, normal; 1 point, renal tubular injury in $<25 \%$; 2 points, renal tubular injury in $25-49 \%$; and 3 points, renal tubular injury in $\geq 50 \%$. Renal interstitial fibrosis classification was as follows: 0 points, normal; 1 point, renal interstitial fibrosis in $<25 \%$ of the field area; 2 points, renal interstitial fibrosis in $25-49 \%$ of the field area; 3 points, renal interstitial fibrosis in $50-74 \%$ of the field area; and 4 points, renal interstitial fibrosis in $\geq 75 \%$ of the field area. The average values of 25 fields were calculated to obtain the renal tubular injury index and renal interstitial fibrosis scores of a specimen.

Detection of the percentage of renal tubular epithelial cell apoptosis using TUNEL staining. After the paraffin sections of the renal tissues were deparaffinized and rehydrated, the procedures were performed according to the instruction manual of the TUNEL reagent kit (Roche Applied Science, Madison, WI, USA). Sections were developed using DAB, and the nuclei were counter stained using hematoxylin. Sections were mounted and observed under a light microscope. Twenty-five different fields were randomly selected under high-power magnification (x400) to count the number of apoptotic renal tubular epithelial cells. The average value was used as the value of apoptosis.

Immunohistochemistry and semi-quantitative analysis. Paraffin-embedded tissue sections of $4-\mu \mathrm{m}$ thickness were sequentially processed with tissue fixation, dehydration and antigen retrieval. Changes in the distribution and expression of Bax, Bcl-2, caspase-3 in the renal tissues were detected using indirect immunostaining. The mouse anti-rat $\mathrm{Bax}, \mathrm{Bcl}-2$ and caspase-3 monoclonal antibodies (1:100; BD Pharmingen, San Diego, CA, USA) were added separately and incubated in a moisture box at $4^{\circ} \mathrm{C}$ overnight. PBS was used to replace the primary antibody and used as the negative control. Horseradish peroxidase-labeled anti-mouse secondary antibody $\operatorname{IgG}(1: 100$; Santa Cruz Biotechnology, Inc., Dallas, TX, USA) was added and incubated in a moisture box at room temperature for $2 \mathrm{~h}$. The results were developed using $\mathrm{DAB}$, and sections were mounted and observed under a microscope. Classification of the Bax, Bcl-2 and caspase- 3 staining was as follows: 0 points, no renal tubular and interstitial staining; 1 point, $<25 \%$ renal tubular staining with a lighter color; 2 points, $25-49 \%$ renal tubular staining with a proper color; 3 points, $50-74 \%$ renal tubular staining with a darker color; and 4 points, $\geq 75 \%$ renal tubular staining with a very dark color. Twenty-five different fields were randomly selected under high-power magnification (x200), and calculations were performed according to the above semi-quantitative method. The average value was the experimental value of the specimen.

Statistical analysis. All data are expressed as the mean \pm standard deviation $(\mathrm{x} \pm \mathrm{s})$. SPSS 17.0 statistical software (SPSS Inc., Chicago, IL, USA) was used for the statistical analysis. Comparison of the mean values between the groups was performed using one-way analysis of variance (one-way ANOVA). Pairwise comparison between the mean values was performed using a t test. $\mathrm{P}<0.05$ indicated the difference had statistical significance.

\section{Results}

Scr and BUN results. The rat Scr and BUN levels were within the normal ranges in all groups, and there was no significant difference among them $(\mathrm{P}>0.05)$ (Fig. 1). 

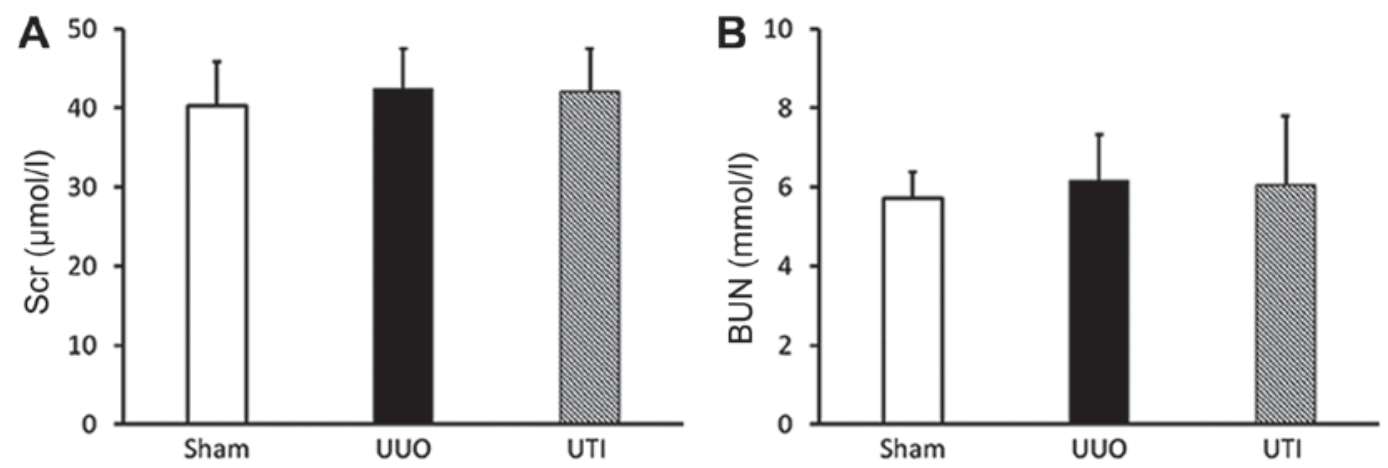

Figure 1. The rat (A) Scr and (B) BUN levels in the Sham, UUO and UTI groups were all within the normal ranges. Comparison among the 3 groups did not show any significant difference $(\mathrm{P}>0.05)$. Scr, serum creatinine; BUN, blood urea nitrogen; UUO, unilateral ureteral obstruction; UTI, ulinastatin.

A

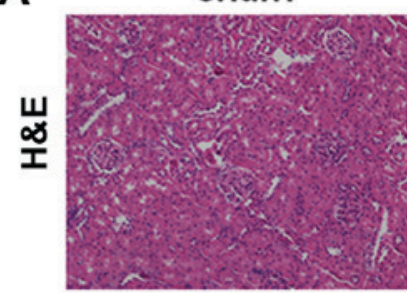

B

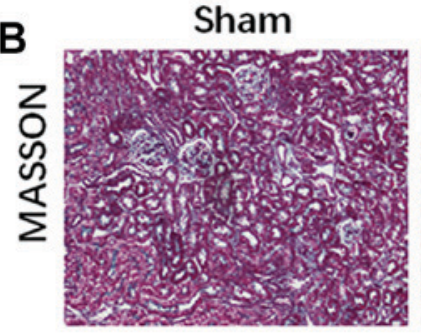

C

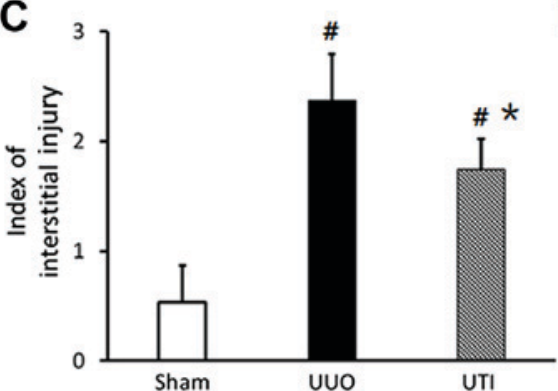

UUO

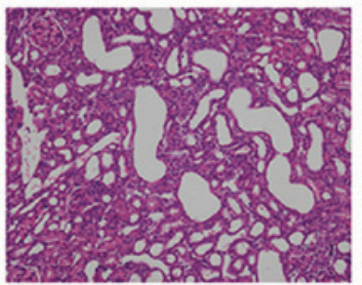

UUO

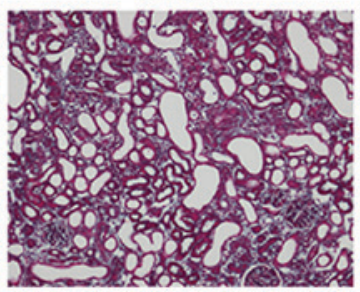

D

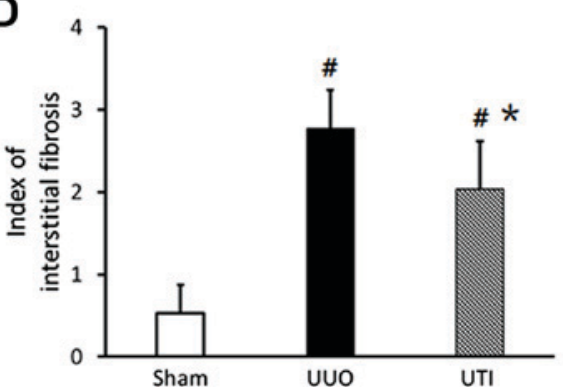

Figure 2. UTI attenuated renal pathological changes of tubulointerstitial injury after ureteral obstruction. (A) H\&E staining results in the Sham, UUO and UTI groups (magnification, x100). (B) Masson staining results in the Sham, UUO and UTI groups (magnification, x100). (C) Statistical graph showing the semi-quantitative analysis of the tubulointerstitial injury results. (D) The statistical graph showing the semi-quantitative analysis of the tubulointerstitial fibrosis results. All data are expressed as the mean \pm standard deviation. Each group comprised 6 rats. ${ }^{*} \mathrm{P}<0.01$ for the UUO and UTI groups compared to the Sham group; ${ }^{*} \mathrm{P}<0.05$ for the UTI group compared to the UUO group. H\&E, hematoxylin and eosin; UUO, unilateral ureteral obstruction; UTI, ulinastatin.

Renal pathological changes. Observation using the naked eye showed that the size and morphology of the kidneys in the Sham group were normal and that the color was ruddy. In the UUO group, the kidney at the obstruction side had obvious swelling and enlargement, the renal pelvis had a large amount of effusion, and the renal parenchyma became thinner. In the UTI group, the kidney had slight swelling and enlargement, there was a small amount of effusion, and the parenchyma became slightly thinner. Under a light microscope, the renal interstitium and renal tubular structure were basically clear and intact, the vacuolar degeneration of renal tubular epithelial cells was observed sporadically, and there was focal inflammatory cell infiltration. The UUO and UTI groups had different degrees of diffuse renal interstitial inflammatory cell infiltration, there was interstitial widening, there were different degrees of accompanied renal tubular dilation or atrophy, there was interstitial fibrosis, and there were no obvious glomerular lesions. The lesions in the UTI group were significantly decreased compared to those in the UUO group. The kidney $\mathrm{H} \& \mathrm{E}$ and Masson staining results in the 3 groups are shown in Fig. 2A and B, and the semi-quantitative scores are shown in Fig. 2C and D. 

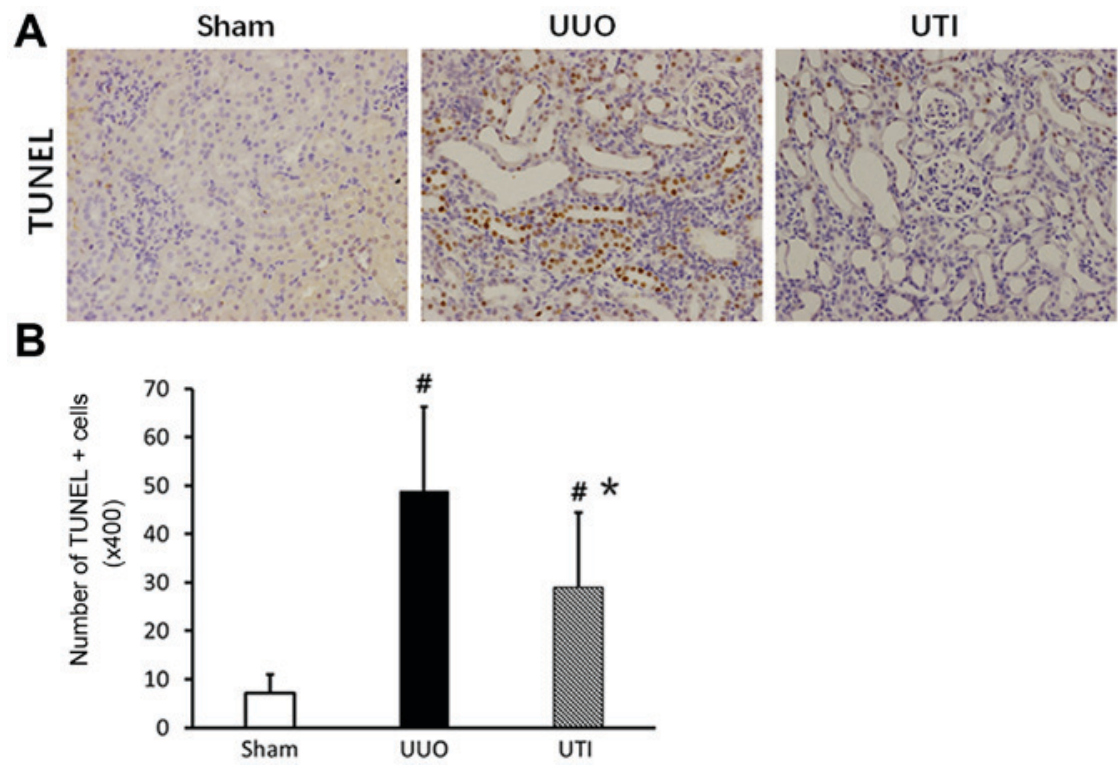

Figure 3. UTI decreased the number of apoptotic cells in the UUO model. (A) The numbers of apoptotic renal tubular epithelial cells in the Sham, UUO and UTI groups (magnification, $\mathrm{x} 400$ ). (B) Statistical graph showing the statistical analysis results of the numbers of apoptotic cells in the 3 groups. All data are expressed as the mean \pm standard deviation. Each group comprised 6 rats. ${ }^{~} \mathrm{P}<0.01$ for the UUO and UTI groups compared to the Sham group; ${ }^{*} \mathrm{P}<0.05$ for the UTI group compared to the UUO group. UUO, unilateral ureteral obstruction; UTI, ulinastatin.

Changes in renal tubular epithelial cell apoptosis. The TUNEL staining results showed that renal tubular epithelial cell apoptosis was sporadically observed in the Sham group, while apoptotic cells in the UUO group significantly increased and were mainly distributed in dilated or atrophic renal tubules. However, compared to that in the UUO group, the number of apoptotic cells in the UTI group significantly decreased. The corresponding results are shown in Fig. 3.

Expression of Bcl-2/Bax in renal tubular epithelial cells. There was more Bcl-2 expression in normal kidney, especially in the cytoplasm of renal tubular epithelial cells. After the UUO injury, the Bcl-2 expression significantly decreased. After the UTI intervention, the expression increased. In the Sham group, the expression of Bax was lower. After UUO, Bax was mainly expressed in dilated or atrophic renal tubules; compared to that in the Sham group, the expression significantly increased. After the UTI, Bax expression was significantly downregulated. The staining results are shown in Fig. 4A and B, and the semi-quantitative scores are shown in Fig. 4C and D.

Caspase-3 expression in renal tubular epithelial cells. Positive caspase- 3 expressing renal tubular epithelial cells mainly localized in dilated or atrophic renal tubules. The cytoplasm of positive cells were yellow or brown, and the cell nuclei presented with partial yellow or brown-yellow color. In the Sham group, the caspase- 3 expression level was lower; in comparison, the expression in the UUO group significantly increased. After UTI treatment, the expression was downregulated. The staining results are shown in Fig. 5A, and the semi-quantitative scores are shown in Fig. 5B.

\section{Discussion}

Renal interstitial fibrosis is a common presentation in all types of kidney diseases of end-stage disease progression. Ureteral obstruction is also an important factor that causes renal interstitial fibrosis. The UUO rat model features include that it does not influence the blood pressure and blood lipid levels of model animals, there is no proteinuria, there is no obvious immune and toxic renal injury, and it does not cause renal impairment (compensated by the contralateral kidney) (18). It is a classical animal model used to study renal interstitial fibrosis (2). Its renal injury process is the result of the common action of many factors, such as various types of cells and cytokines. However, the question of how to block and/or reverse the renal interstitial fibrosis process is of significant clinical concern.

UTI is a glycoprotein isolated and purified from human urine. It has inhibitory functions on many types of enzymes, including serine proteases such as trypsin and $\alpha$-chymotrypsin, granulocyte elastase, hyaluronidase, thiolase and plasmin (6), playing a role in the clearance of oxygen free radicals and inhibition of release of inflammatory mediators and neutrophil activation (7). It is used clinically for patients with acute pancreatitis, ischemia/reperfusion injury, multiple organ dysfunction syndrome (MODS), acute respiratory distress syndrome (ARDS), organ transplantation, post-cardiopulmonary bypass surgery, inhibition of apoptosis, inhibition of inflammation, protection of cells and improvement of circulation and coagulation. UTI also has immune regulation functions; it can attenuate excessive inflammatory reactions and protect lung injury induced by lipopolysaccharide (LPS) and severe burns (7). Animal studies have already indicated that UTI significantly increased superoxide dismutase (SOD) in the renal tissues of renal injury rats induced by severe burns and decreased Scr and BUN (11). UTI attenuated renal tubular epithelial cell necrosis, inflammatory cell infiltration and BUN increase in yeast polysaccharide-induced multiple organ dysfunction in rats (12). The biochemical detection results in this study showed that neither UUO nor UTI significantly affected renal function in experimental rats. The H\&E and Masson staining results showed that after UTI treatment, the 
A

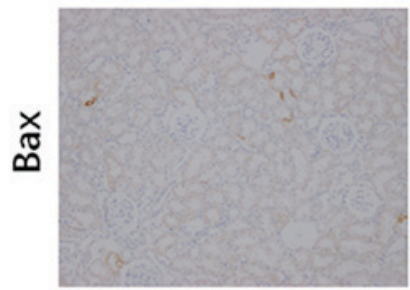

B

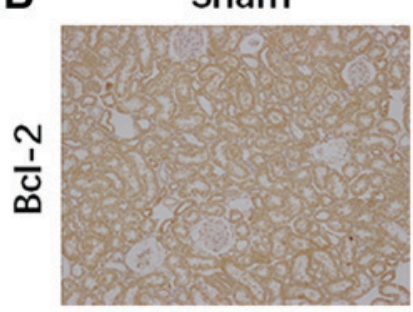

C

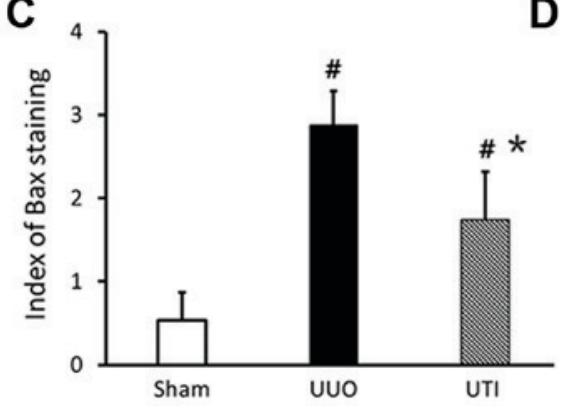

D
UUO

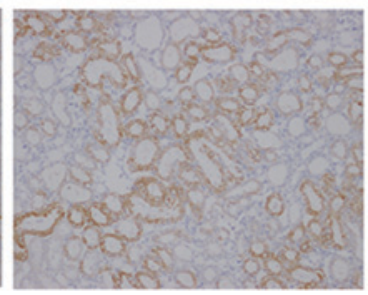

UUO
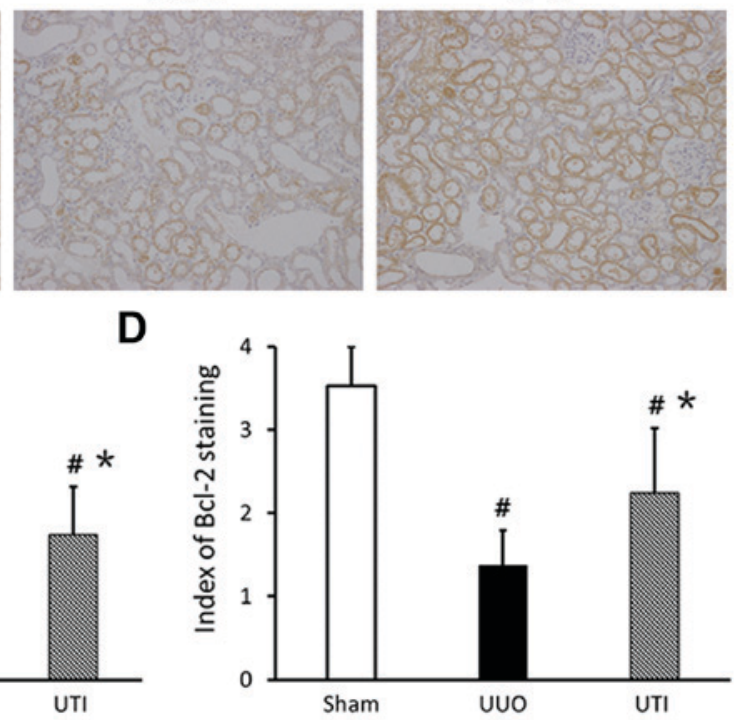

Figure 4. Bax expression decreased and Bcl-2 expression increased in the UUO model after UTI intervention. (A) Expression of Bax in the Sham, UUO and UTI groups (magnification, x200). (B) Expression of Bcl-2 in the Sham, UUO and UTI groups (magnification, x200). (C) Statistical graph showing the semi-quantitative analysis of Bax expression in the 3 groups. (D) Statistical graph showing the semi-quantitative analysis of Bcl-2 expression in the 3 groups. All data are expressed as the mean \pm standard deviation. Each group comprised 6 rats. ${ }^{~} \mathrm{P}<0.01$ for the UUO and UTI groups compared to the Sham group; ${ }^{*} \mathrm{P}<0.05$ for the UTI group compared to the UUO group. UUO, unilateral ureteral obstruction; UTI, ulinastatin; Bax, apoptosis regulator Bax; Bcl-2, apoptosis regulator Bcl-2.

A

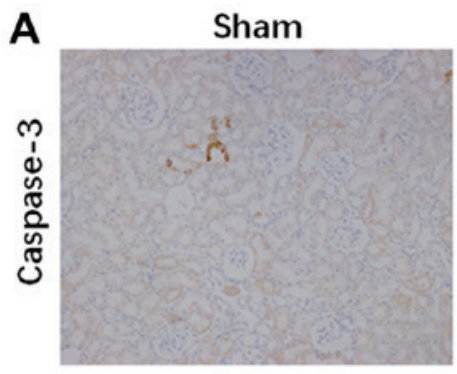

B

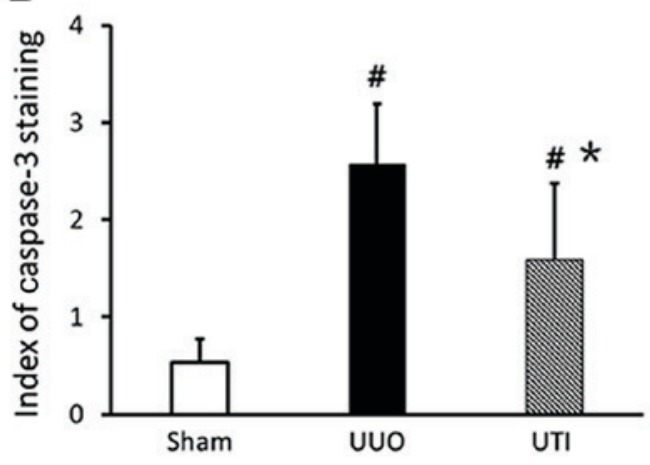

Figure 5. UTI decreased caspase-3 expression in the UUO model. (A) Expression of caspase-3 in the Sham, UUO and UTI groups (magnification, x200). (B) Statistical graph showing the semi-quantitative analysis of caspase-3 expression in the 3 groups. All data are expressed as the mean \pm standard deviation. Each group comprised 6 rats. ${ }^{~} \mathrm{P}<0.01$ for the UUO and UTI groups compared to the Sham group; ${ }^{*} \mathrm{P}<0.05$ for the UTI group compared to the UUO group. UUO, unilateral ureteral obstruction; UTI, ulinastatin. 
renal interstitial injury index of the UUO rats significantly decreased and the area of fibrosis significantly decreased, which was similar to previous results (14). These results indicated that UTI had a protective function in obstructive renal tissue injury.

UTI has been shown to inhibit lung fibrosis (13) and renal fibrosis through blocking the TGF- $\beta /$ Smad signaling pathway (14). Excessive proliferation of myoblasts and excessive accumulation of extracellular matrix (including COL-I, CIL-III and fibronectin) are the major pathological changes in renal interstitial fibrosis. $\alpha$-SMA is a marker of myoblasts. There are studies showing that after UUO, the rat renal tissues had significantly increased COL-I and $\alpha$-SMA expression and exhibited renal interstitial fibrosis. Compared to the UUO group, COL-I and $\alpha$-SMA significantly decreased after UTI treatment, renal interstitial extracellular matrix decreased, and renal interstitial fibrosis significantly decreased $(14,19)$. These results indicate that UTI had a specific function in the resistance to fibrosis in obstructive renal tissue injury.

There are many factors causing apoptosis in ureteral obstruction, such as ischemia and hypoxia, cytokines, growth factors, release of reactive oxygen and mechanical stretch (20). A large number of studies have indicated that excessive apoptosis decreased the number of renal tubular epithelial cells to cause renal tubular atrophy, thus accelerating the progression of renal interstitial fibrosis (21). Inhibition of cell apoptosis may delay or reverse renal tubular interstitial fibrosis, thus improving the renal function and prognosis of patients with kidney diseases (4). In addition, recent studies indicated that apoptosis in the hippocampus of gerbils increased after cerebral ischemia, while UTI treatment inhibited Bax molecule expression and increased $\mathrm{Bcl}-2$ expression in ischemia-induced apoptosis in hippocampus and decreased the TUNEL+ and caspase-3+ cells in the hippocampal CA1 region induced by cerebral ischemia, thus improving ischemia-induced short-term memory impairment (9). UTI reduced the heatstroke-induced $\mathrm{Bax} / \mathrm{Bcl}-2$ ratio and caspase- 3 levels in brain cells and inhibited cell apoptosis during cerebral injury (10). In this study, the TUNEL results showed that obvious renal tubular epithelial cell apoptosis occurred after 7 days of UUO surgery. Compared to the UUO group, renal interstitial cell apoptosis significantly decreased after UTI treatment.

The Bcl-2 family and the caspase family members are major signaling regulatory proteins in cell apoptosis pathways. Currently, $25 \mathrm{Bcl}-2$ family homologous proteins have been discovered. Some members, such as Bad, Bid and Bax, have apoptosis promotion functions, and some members, such as Bcl-2, Bcl-x and Bcl-w, can block the release of cytochrome C from mitochondria to cytoplasm to inhibit cell apoptosis (22). The caspase family member caspase- 3 is the most important terminal (PARP) cleavage enzyme. It is an apoptosis executor and is also an important component in the killing mechanism of CTL.

The immunohistochemistry results in this study indicated that compared to those in the Sham group, Bax and caspase-3 expression increased and Bcl-2 expression decreased in the renal tubular epithelium of rats in the UUO group. UUO increased the Bax/Bcl-2 ratio and caspase- 3 levels to induce cell apoptosis. Compared to that in the UUO group, Bax and caspase-3 expression significantly decreased, and Bcl-2 expression was upregulated in the UTI treatment group. These results indicated that UTI reduced cell apoptosis through the regulation of their expression levels.

In summary, UTI inhibited renal tubular epithelial cell apoptosis and fibrosis in UUO rats. These results suggest that UTI may play a role in preventing renal interstitial fibrosis through the inhibition of renal tubular epithelial cell apoptosis; therefore, UTI demonstrated a protective function against renal injury on the obstruction side. As an endogenous protease inhibitor, UTI has complicated biological functions. Its protective mechanism in UUO renal injury merits further study.

\section{Acknowledgements}

The present study was supported by the Scientific Research Project of the Department of Health of Heilongjiang Province of China (2010-099) and the Youth Fund Project of the Second Affiliated Hospital of Harbin Medical University (QN2011-21).

\section{References}

1. Liu Y: Renal fibrosis: New insights into the pathogenesis and therapeutics. Kidney Int 69: 213-217, 2006.

2. Chevalier RL, Forbes MS and Thornhill BA: Ureteral obstruction as a model of renal interstitial fibrosis and obstructive nephropathy. Kidney Int 75: 1145-1152, 2009.

3. Chevalier RL, Thornhill BA, Forbes MS and Kiley SC: Mechanisms of renal injury and progression of renal disease in congenital obstructive nephropathy. Pediatr Nephrol 25: 687-697, 2010.

4. Docherty NG, O'Sullivan OE, Healy DA, Fitzpatrick JM and Watson RW: Evidence that inhibition of tubular cell apoptosis protects against renal damage and development of fibrosis following ureteric obstruction. Am J Physiol Renal Physiol 290: F4-F13, 2006.

5. Letai A: Pharmacological manipulation of Bcl-2 family members to control cell death. J Clin Invest 115: 2648-2655, 2005.

6. Fries E and Blom AM: Bikunin-not just a plasma proteinase inhibitor. Int J Biochem Cell Biol 32: 125-137, 2000.

7. Fang $\mathrm{Y}, \mathrm{Xu} \mathrm{P}, \mathrm{Gu} \mathrm{C}$, Wang $\mathrm{Y}, \mathrm{Fu} \mathrm{XJ}, \mathrm{Yu} \mathrm{WR}$ and Yao M: Ulinastatin improves pulmonary function in severe burn-induced acute lung injury by attenuating inflammatory response. J Trauma 71: 1297-1304, 2011.

8. Xu M, Wen XH, Chen SP, An XX and Xu HY: Addition of ulinastatin to preservation solution promotes protection against ischemia-reperfusion injury in rabbit lung. Chin Med J (Engl) 124: 2179-2183, 2011.

9. Cho YS, Shin MS, Ko IG, Kim SE, Kim CJ, Sung YH, Yoon HS and Lee BJ: Ulinastatin inhibits cerebral ischemia-induced apoptosis in the hippocampus of gerbils. Mol Med Rep 12: 1796-1802, 2015.

10. Tao Z, Hu FQ, Li CF, Zhang T, Cao BZ and Cui LQ: Effect of ulinastatin, a human urinary protease inhibitor, on heatstroke-induced apoptosis and inflammatory responses in rats. Exp Ther Med 13: 335-341, 2017.

11. Gao C, Huan J, Li W and Tang J: Protective effects of ulinastatin on pancreatic and renal damage in rats following early scald injury. Burns 35: 547-552, 2009.

12. Yang Q, Liu X, Liu M,Zhang L and Guan Y: Ulinastatin-mediated protection against zymosan-induced multiple organ dysfunction in rats. Biologicals 38: 552-556, 2010.

13. Katoh H, Ishikawa H, Hasegawa M, Yoshida Y, Suzuki Y, Ohno T, Takahashi T and Nakano T: Protective effect of urinary trypsin inhibitor on the development of radiation-induced lung fibrosis in mice. J Radiat Res 51: 325-332, 2010.

14. Ning XH, Ge XF, Cui Y and An HX: Ulinastatin inhibits unilateral ureteral obstruction-induced renal interstitial fibrosis in rats via transforming growth factor $\beta$ (TGF- $\beta$ )/Smad signalling pathways. Int Immunopharmacol 15: 406-413, 2013.

15. National Research Council (NRC): Guide for the Care and Use of Laboratory Animals. 8th edition. National Academies Press, Washington, DC, 2011. 
16. Satoh M, Kashihara N, Yamasaki Y, Maruyama K, Okamoto K, Maeshima Y, Sugiyama H, Sugaya T, Murakami K and Makino H: Renal interstitial fibrosis is reduced in angiotensin II type 1a receptor-deficient mice. J Am Soc Nephrol 12: 317-325, 2001.

17. Katafuchi R, Kiyoshi Y, Oh Y, Uesugi N, Ikeda K, Yanase T and Fujimi S: Glomerular score as a prognosticator in IgA nephropathy: Its usefulness and limitation. Clin Nephrol 49: 1-8, 1998

18. Grande MT, Pérez-Barriocanal F and López-Novoa JM: Role of inflammation in túbulo-interstitial damage associated to obstructive nephropathy. J Inflamm (Lond) 7: 19, 2010.

19. Jiang GT, Chen X, Dong L, An HX and Jiao JD: Ulinastatin attenuates renal interstitial inflammation and inhibits fibrosis progression in rats under unilateral ureteral obstruction. Mol Med Report 10: 1501-1508, 2014.
20. Misseri R and Meldrum KK: Mediators of fibrosis and apoptosis in obstructive uropathies. Curr Urol Rep 6: 140-145, 2005.

21. Maoka T, Tokuda H, Suzuki N, Kato $\mathrm{H}$ and Etoh $\mathrm{H}$ : Anti-oxidative, anti-tumor-promoting, and anti-carcinogensis activities of nitroastaxanthin and nitrolutein, the reaction products of astaxanthin and lutein with peroxynitrite. Mar Drugs 10: 1391-1399, 2012.

22. Dolka I, Król M and Sapierzyński R: Evaluation of apoptosis-associated protein (Bcl-2, Bax, cleaved caspase-3 and p53) expression in canine mammary tumors: An immunohistochemical and prognostic study. Res Vet Sci 105: 124-133, 2016. 